\title{
The four-quadrant phase-mask coronagraph: white light laboratory results with an achromatic device
}

\author{
D. Mawet ${ }^{1}$, P. Riaud ${ }^{1}$, J. Baudrand ${ }^{2}$, P. Baudoz ${ }^{2}$, A. Boccaletti ${ }^{2}$, O. Dupuis ${ }^{2}$, and D. Rouan ${ }^{2}$ \\ 1 Université de Liège, 17 Allée du 6 Août, 4000 Sart-Tilman, Belgium \\ 2 LESIA, Observatoire de Paris-Meudon, 5 pl J. Janssen, 92195 Meudon, France \\ e-mail: mawet@astro.ulg.ac.be
}

Received 6 September 2005/ Accepted 8 November 2005

ABSTRACT

Achromatic coronagraphs are the subject of intensive research since they will be mandatory for many programs which aim at detecting and characterizing exoplanets. We report a laboratory experiment assessing the performance of the Four-Quadrant Phase-Mask coronagraph (FQPM) over a broadband wavelength range $(R \approx 2)$. The achromatization of the FQPM is provided by achromatic halfwave plates (HWP). These phase shifters combine birefringent plates made of different materials with properly chosen thicknesses. The HWP thickness control is relaxed by two orders of magnitudes with respect to the classical (non-birefringent) dispersive plate approach. In our experiment we used a two stage stack of Quartz and $\mathrm{MgF}_{2}$. This combination allows to cover a large spectral range in the visible (500-900 nm) with a small phase error residual around $\pi(\approx 0.12 \mathrm{rad} \mathrm{rms})$. With this achromatization, we obtained an attenuation of 755 on the white light PSF peak. This solution is directly applicable to ground-based telescopes using high order adaptive optics such as the ESO's VLT-Planet Finder project and could easily be transposed in the mid-infrared domain for future space-based missions like DARWIN/TPF.

Key words. instrumentation: adaptive optics - techniques: high angular resolution - stars: planetary systems - methods: observational

\section{Introduction}

Direct detection of faint sources around bright astrophysical objects such as exoplanets orbiting their parent star is very difficult due to the large flux ratio. For example, extrasolar planets are typically $10^{4}-10^{7}$ times fainter than their host star in the infrared. Therefore, the study of such objects necessitates coronagraphic instruments and nearly perfect wavefronts for optimal operations. Subsequently, this challenging science case requires:

- for Extrasolar Earth-like planets, dedicated space-based observatories like the DARWIN/TPF projects (see Léger et al. 1996, for instance);

- for Extrasolar Giant Planets (EGPs), space-based observatories or Extreme-Adaptive Optics ground-based imaging facilities, like the ESO's VLT-Planet Finder (Mouillet et al. 2003).

A few years ago, we proposed a new device, the Four-Quadrant Phase-Mask coronagraph (FQPM), to advantageously replace Lyot-type amplitude coronagraphs. The FQPM uses a four quadrant $\pi$ phase shift distribution in the focal plane to provide a self-destructive interference for a centered point like monochromatic source. The FQPM provides significantly smaller working distances than Lyot-type coronagraphs as well as better rejection factors at high Strehl ratios. After presenting the principles and an extensive numerical study (Rouan et al. 2000; Riaud et al. 2001), a third paper (Riaud et al. 2003) reported the laboratory results with a real component in monochromatic light. Since then, the actual performance of the setup has been largely improved: we have obtained a very stable peak attenuation of $10^{5}$ and a total rejection factor of $10^{4}$. The current setup only works accurately for a single wavelength. Observing with a large bandwidth like the $K$ band (2-2.4 $\mu \mathrm{m})$ with a monochromatic mask would yield a theoretical rejection of only 150 . However, for ground-based telescopes using today's state of the art adaptive optics, such as NACO (VLT's NAOS-CONICA, Rousset et al. 1998), a monochromatic phase mask is sufficient for the full $K$ band, since the main limitation comes from the residual uncompensated wavefront errors (Boccaletti et al. 2004).

For higher order corrections such as planned for the ESO's VLT-Planet Finder second generation instrument on the VLT, the chromaticity issue of the FQPM is no longer negligible. Moreover, this instrument requires multi-wavelength operations $(0.9-2.5 \mu \mathrm{m})$. An achromatic device is thus needed. We therefore propose to implement commercially available achromatic halfwave plates (HWP) in an original way to reproduce the particular FQPM focal plane phase shift distribution. Section 2 describes the principle of birefringent achromatic retarders and presents numerical simulations to assess theoretical 


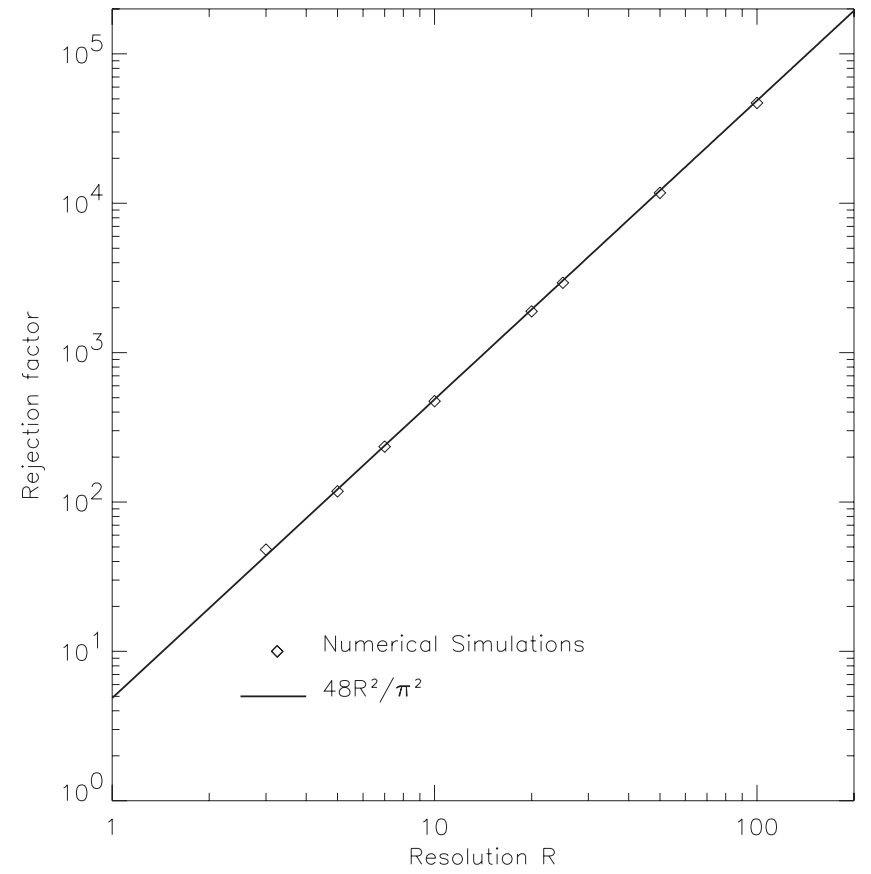

Fig. 1. Rejection $\tau$ of a monochromatic component as a function of the spectral resolution $R$. The continuous line is for the analytical formula whereas the diamonds show coronagraphic numerical simulation results.

limitations of this technique regarding the total rejection. In Sect. 3, after reviewing some manufacturing issues, we present the results of our white light coronagraphic experiment. We discuss in Sect. 4 the possibility of extending this technique to other wavelength ranges, like the mid-infrared domain. We also discuss a very promising technique using synthetic birefringence created on a unique substrate made of any transparent material.

\section{Principle}

Before presenting the principle of an achromatic FQPM, let us first define the metrics we shall use (Boccaletti et al. 2004). A coronagraph's ability to suppress the on-axis starlight can be quantified with two different parameters:

- the total rejection: ratio of total intensity of the direct image to that of the coronagraphic image;

- the peak attenuation: ratio of the maximum (on-axis) intensity of the direct image to that of the coronagraphic image.

It is important to note that these metrics are not always related in the same way according to the working conditions.

\subsection{Monochromatic phase masks}

In the past, our team only manufactured monochromatic phase masks using thin film deposition or etching techniques based on the "index step" principle: a step of height $h$ in a material of refractive index $n$ at the wavelength $\lambda$ induces a phase shift

$\Delta \phi=\frac{2 \pi}{\lambda}(n-1) h$
The hyperbolic phase shift dependence in $\lambda$ makes the technique unapplicable for high performance use over a large spectral bandwidth. In fact, it was shown (Riaud et al. 2003) that the broadband total rejection for a monochromatic mask is

$\tau \approx \frac{48}{\pi^{2}} R^{2}$

where $R=\lambda / \Delta \lambda$ is the spectral resolution. Figure 1 shows the total rejection expected for a monochromatic component with respect to the spectral resolution. We clearly notice its rapid deterioration in the broadband cases. For example, in the 500-900 nm wavelength range $(R=1.75)$, the highest total rejection expected with a monochromatic mask is only $\tau=15$. In this case, the coronagraphic numerical simulation gives a peak attenuation of 35 (see Sect. 3.4).

In order to detect and characterize young EGPs, next generation AO instruments like the ESO's VLT-Planet Finder will require larger stellar rejections for several broadband filters (from $J$ to $K$ ). Numerical simulation in $H$ band assuming a Strehl ratio of $80 \%^{1}$ gave a coronagraphic peak attenuation of about 450 . This result was obtained assuming a phase shift error with respect to $\pi$ of $0.01 \mathrm{rad}$. This value can be considered as a specification on the mask chromatic residuals since the same simulation with a 0.1 rad error showed an evident degradation of the peak attenuation of about 3.5 after speckle calibration. A specific broadband coronagraph is therefore necessary. Achromatic waveplates are the solution we consider in this paper.

\subsection{Achromatic waveplates}

Waveplates are optical elements which introduce a phase shift between the polarization components $s$ and $p$ of the incident light. Most of them use the birefringence phenomenon. Birefringence is a natural property of anisotropic crystals but can also be artificially created using one-dimensional subwavelength gratings (Mawet et al. 2005b). The birefringence is defined as follows

$\Delta n=n_{\mathrm{e}}-n_{\mathrm{o}}$

where $n_{\mathrm{o}}$ and $n_{\mathrm{e}}$ are the ordinary and extraordinary indices associated with the two privileged directions of vibration, $s$ and $p$. If the birefringence $\Delta n$ is negative, the medium is said "negative uniaxial". In this case, one defines the fast (resp. slow) axis as the axis associated with the extraordinary (resp. ordinary) index. Conversely, if the birefringence is positive, the medium is said "positive uniaxial". In this case, the fast (resp. slow) axis is defined as the axis associated with the ordinary (resp. extraordinary) index.

Achromatic waveplates (halfwave, quarterwave,...) are commonly produced by combining two plates of different birefringent materials with properly chosen thicknesses. Since the dispersion of the birefringence is different for the two materials, it is possible to make Optical Path Difference (OPD) values linear with $\lambda$ within a given wavelength range. Hence,

\footnotetext{
${ }^{1}$ Expected performance for the 1340 actuators of the VLT-PF Adaptive Optics.
} 

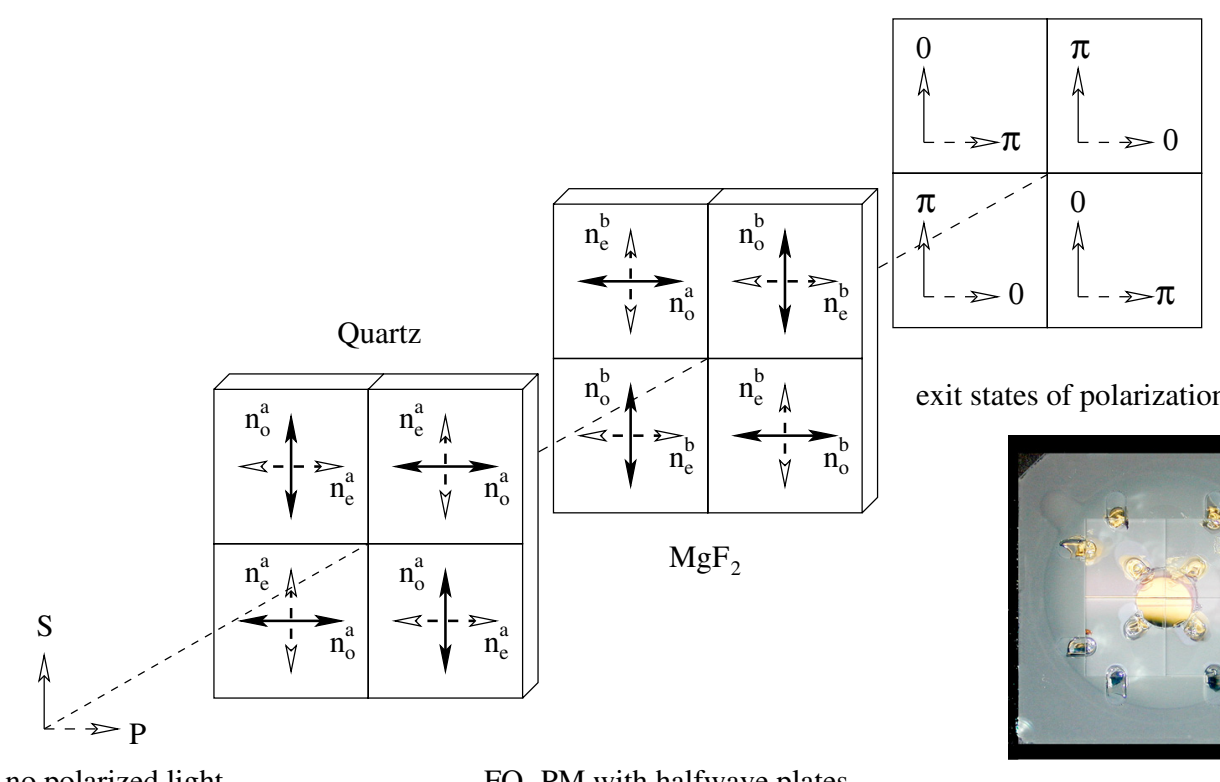

exit states of polarization

no polarized light

FQ-PM with halfwave plates

Fig. 2. Principle of a FQPM using halfwave plates. Each individual quadrant consists of a two material stack $\left(\mathrm{Quartz}_{\mathrm{MgF}}\right)$. Two quadrants along one diagonal are rotated by $90^{\circ}$ around their normals with respect to the two others. The subscript a and $\mathrm{b}$ stand for the Quartz and $\mathrm{MgF}_{2}$ plates whereas the o and e ones, for the ordinary and extraordinary indices, respectively. The bottom right picture shows the final component assembled on a silicate substrate. Fixation points (optical glue) are clearly visible at the waveplate external edges. The $8 \mathrm{~mm}$-diameter circular working area is at the center of the component.

the retardation of the resulting waveplate can be made little sensitive to the wavelength (Hariharan 1996). We consider a combination of two such birefringent plates of thicknesses $d_{\mathrm{a}}$ and $d_{\mathrm{b}}$ and of birefringences $\Delta n_{\mathrm{a}}$ and $\Delta n_{\mathrm{b}}$. The usual condition for achromatism is that the retardation of the system $\Delta \phi=\frac{2 \pi}{\lambda}\left(d_{\mathrm{a}} \Delta n_{\mathrm{a}}+d_{\mathrm{b}} \Delta n_{\mathrm{b}}\right)$ should be equal to half a wave (for a halfwave retarder) at two selected wavelengths $\lambda_{2}$ and $\lambda_{3}$ while minimizing the phase shift error with respect to the chosen retardation value over the $\lambda_{1}-\lambda_{4}$ wavelength range. Therefore we have

$d_{\mathrm{a}} \Delta n_{\mathrm{a}}\left(\lambda_{2}\right)+d_{\mathrm{b}} \Delta n_{\mathrm{b}}\left(\lambda_{2}\right)=\frac{\lambda_{2}}{2}$
$d_{\mathrm{a}} \Delta n_{\mathrm{a}}\left(\lambda_{3}\right)+d_{\mathrm{b}} \Delta n_{\mathrm{b}}\left(\lambda_{3}\right)=\frac{\lambda_{3}}{2}$

$\epsilon=\min \int_{\lambda_{1}}^{\lambda_{4}}|\Delta \phi(\lambda)-\pi|^{2} \mathrm{~d} \lambda$

where $\Delta n_{\mathrm{a}}\left(\lambda_{2}\right), \Delta n_{\mathrm{b}}\left(\lambda_{2}\right), \Delta n_{\mathrm{a}}\left(\lambda_{3}\right)$ et $\Delta n_{\mathrm{b}}\left(\lambda_{3}\right)$ are, respectively, the values of the birefringence of the two materials at these two wavelengths. A solution for Eqs. (4)-(6) can be obtained by combining a material with a positive birefringence with one having a negative birefringence. Conversely, two materials whose birefringences have the same sign can be combined if their fast axis are set perpendicular. Let us mention the special case of the so-called "superachromatic" retarder (Frecker et al. 1976): it consists of three pairs of two materials. This configuration is very performing but is bulky and would therefore induce a huge defocus error and a ghost problem (12 interfaces).

The interest in using naturally birefringent phase shifters and a reason why they are commercially available comes from the fact that, usually, the birefringence is two orders of magnitudes smaller than the indices. So a thickness error contributes to the phase shift two orders of magnitude less. On the other hand, we need very accurate measurements of the birefringence for the used materials, over the range of wavelengths to be covered. This is generally the case for commercially available devices.

\subsection{FQPM with waveplates}

This section explains how the concept of polarization phase shifters is adapted to the FQPM. Let $s$ and $p$ be the global polarization components of the incoming light. In each of the four quadrants made of cut halfwave plates, the $s$ and $p$ global polarization states are projected according to the local fast and slow axis orientations of the two-level stack. We have already seen that two indices, the ordinary index $n_{\mathrm{o}}$ and the extraordinary index $n_{\mathrm{e}}$, can be assigned to these directions. Let us now assume that the four cut quadrants are strictly identical (same two-level stack) and implemented in the following way: two of them in two opposite quadrants along one diagonal are rotated by $90^{\circ}$ around their normals with respect to the two others. This antisymmetrical configuration mimics the FQPM particular focal plane $\pi$-phase distribution for each parallel and potentially interfering state of polarization (see Fig. 2). Such a design works with natural light. In practice, all plates have to be polished and cut in the same material blank to obtain homogenous components in terms of refractive index and surface quality.

Another way to make the FQPM achromatic was proposed and tested by Baba et al. (2002): the polarization interferometric coronagraph. It is in fact a FQPM whose phase shift is provided by a Liquid-Crystal (LC) device sandwiched between two crossed polarizers. This method allows a broadband use 


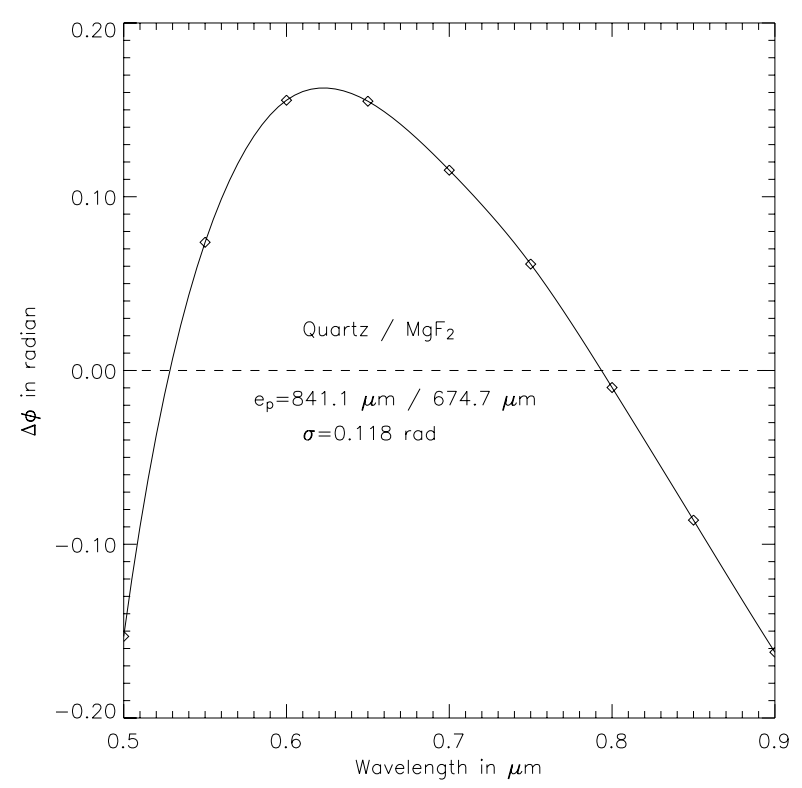

Fig. 3. Numerical simulation result for achromatic halfwave plates. The curve shows the phase residuals around $\pi$ in radian versus wavelength (microns). The diamonds indicate the points where the birefringences have been precisely measured by the manufacturer. The phase error standard deviation over the full wavelength range is $0.118 \mathrm{rad} \mathrm{rms}$.

of the coronagraph, but only with linearly polarized light in the visible.

\subsection{Theoretical performance}

The selected retarder in our experiment is a combination of Quartz $\left(\mathrm{SiO}_{2}\right)$ and $\mathrm{MgF}_{2}$ crystal plates. Quartz and $\mathrm{MgF}_{2}$ are often associated for the good matching of their respective birefringence dispersions. Both are transparent from the ultraviolet to the near infrared, have low indices (low Fresnel losses) and can be easily polished (low wavefront errors). The mean birefringences over the wavelength range $\left(\lambda_{1}(=500 \mathrm{~nm})-\right.$ $\lambda_{4}(=900 \mathrm{~nm})$ ) are respectively $\Delta n_{\mathrm{a}} \approx 0.00900$ for Quartz (positive uniaxial) and $\Delta n_{\mathrm{b}} \approx-0.01190$ for $\mathrm{MgF}_{2}$ (negative uniaxial). Note that birefringence values at the $10^{-5}$ level of precision can be easily found in the literature for these materials. For the working spectral range, we found the following couple of optimized thicknesses: $d_{\mathrm{a}}=841.1 \mu \mathrm{m}$ for Quartz and $d_{\mathrm{b}}=674.7 \mu \mathrm{m}$ for $\mathrm{MgF}_{2}$. In Fig. 3, we present the residual phase shift error obtained for this optimal solution, with a standard deviation $\sigma=0.118 \mathrm{rad}$ rms over the full spectral band. The total rejection for a nulling phase mask coronagraph being related to the phase error standard deviation $\sigma$ by $\tau=\frac{4}{\sigma^{2}}$ (Riaud et al. 2003), we therefore obtain $\tau \approx 290$ assuming phase errors only.

\section{Laboratory results}

\subsection{Achromatic FQPM assembly}

The achromatic phase mask assembly is mounted on a silica substrate $1 \mathrm{~mm}$ thick with a central hole of $8 \mathrm{~mm}$ in diameter.
The four Quartz plates are assembled on one side and the four remaining $\mathrm{MgF}_{2}$ plates on the other one. According to the implementation scheme presented here above, the $\pi$ phase shift between adjacent quadrants is provided by rotating the fast axis of two quadrant stacks along one diagonal by $90^{\circ}$ around their normals. Each of the 8 individual plates is polished $(\lambda / 10$ PTV at $550 \mathrm{~nm}$ ) and cut parallel to the optical axis with a micrometric precision. The edge parallelism cutting error with respect to each plate fast/slow axis is below 30 arcsecs. All plates are anti-reflection coated for the considered bandwidth (reflectivity $<1 \%$ ). One of us (O. D.) assembled the eight plates with a high precision $(<10 \mu \mathrm{m})$ while respecting orientations, alignments and coplanarity ( $<10$ arcmin) for the two stages. This task revealed to be practically difficult and long to achieve within the specifications. For example, the coplanarity issue was overcome only by performing the assembly in a clean environment and under in situ interferometric metrology. Indeed, a $4 \mu \mathrm{m}$ dust particle, for instance, can induce a 14 arcmin outof-plane deviation.

In order to hide the imperfections at the edges resulting from the cutting process, we added a thin opaque spider (a tungsten wire $18 \mu \mathrm{m}$ in diameter) over the four quadrant transitions. Indeed, the rough cut edges at the transitions would otherwise diffract too much light inside the geometric pupil area.

\subsection{Optical setup}

The optical bench is the one used for the FQPM monochromatic tests (see Riaud et al. 2003). The white light source is a halogen lamp at a temperature of $3400 \mathrm{~K}(100 \mathrm{~W})$ and the setup is feeded with a $4 \mu \mathrm{m}$ core fiber (single mode for wavelengths longer than $620 \mathrm{~nm}$ ). All lenses are in silica (infrasil 301) with low frequency surface errors lower than $\lambda / 20$ PTV or $\lambda / 80 \mathrm{rms}$ across $10 \mathrm{~mm}$ in diameter and AR coated for use between 400 and $1100 \mathrm{~nm}$ (reflectivity $<1 \%$ per interface). The entrance pupil is a hole with a diameter of $1.78 \mathrm{~mm}$. In this configuration the $f$-number is 253 and the size of the Airy pattern projected on the achromatic phase mask is $\lambda / d=176 \mu \mathrm{m}$ at $700 \mathrm{~nm}$. This size is one order of magnitude above the precision of the plate adjustment $(\approx 10 \mu \mathrm{m})$. Finally, to filter the coronagraphic diffraction at the edge of the pupil, we used a diaphragm of $350 \mu \mathrm{m}$ in diameter, designed to undersize the pupil by about $23 \%$ in diameter. This size for the Lyot stop (i.e. nearly $80 \%$ ) corresponds to a trade-off between the diffraction residuals filtering and global throughput (see Riaud et al. 2001). The Strehl ratio estimated for the $532 \mathrm{~nm}$ PSF image is $99.8 \%$, which is equivalent to $\lambda / 180 \mathrm{rms}$ for the total wavefront error.

\subsection{Acquisition and data processing}

All images were recorded with a ST8 XE Sbig camera equipped by a KAF 1600 E CCD chip cooled by one Peltier module. The sampling in our configuration $(3 \times 3$ binning $)$ is 43 pixels per $\lambda_{0} / d$, with $\lambda_{0}=700 \mathrm{~nm}$ being the central wavelength of the 500-900 $\mathrm{nm}$ bandpass filter. To assess the broadband coronagraphic performance of the component, we first 


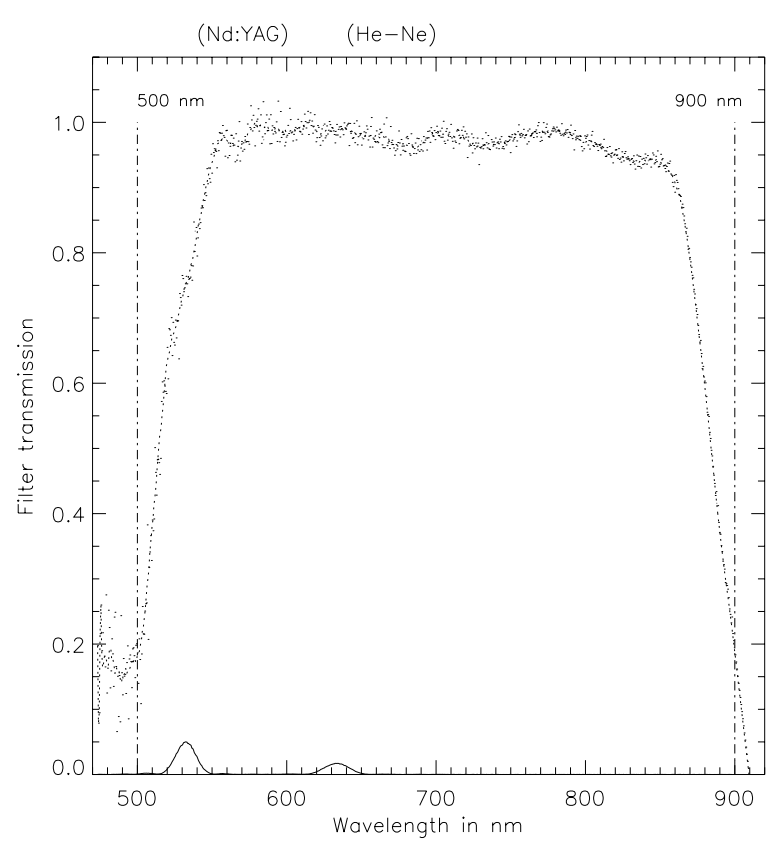

Fig. 4. This figure presents the spectral filter response as measured on our coronagraphic bench. The spectral sampling is $0.55 \mathrm{~nm} / \mathrm{pixel}$. The two peaks below, convolved with the PSF, correspond to calibration lines obtained with a YAG: $\mathrm{Nd}$ and a $\mathrm{He}-\mathrm{Ne}$ laser, respectively. The maximum transmission of the filter is $96 \pm 3 \%$.

acquired a coronagraphic image with the white halogen lamp and the bandpass filter characterized in Fig. 4. The exposure time was about $30 \mathrm{~s}$. Forty exposures were co-added to improve the signal-to-noise ratio up to 1000 . The next step was to record direct non-coronagraphic images in the same conditions but with a shorter exposure time to avoid saturation (1 s). All images were then subtracted with a median dark frame. Results in terms of coronagraphic rejection normalized profiles are displayed in Figs. 5 and 7.

The visible waveplates are optimized for the 500-900 nm wavelength range but they are also quite good for the 700-1000 nm range. Therefore, we have also tested the component with a Schott RG 645 highpass filter with a maximum transmission of $91 \%$. The cutoff at long wavelengths is ensured by the CCD low sensitivity around $1.1 \mu \mathrm{m}$. In Fig. 7, the corresponding coronagraphic rejection profile is also shown.

\subsection{Performance}

The attenuation on the stellar peak is about 755 for the 500-900 nm bandpass (see Fig. 5) and 561 for the $700-1000 \mathrm{~nm}$ one (see Fig. 7). The total rejection is 294 for the first bandpass and 256 for the second one. The results we obtained indicate that the attenuation on the central peak is mostly limited by the mask residual chromatism rather than the surface roughness $\left(\approx \lambda_{0} / 1000 \mathrm{rms}\right)$ or the size of the source. Indeed, before each polychromatic measurements, the optical bench was calibrated with a monochromatic mask and a laser diode source, routinely giving a peak attenuation of about $10^{5}$ (see Riaud et al. 2003). Moreover, the measurements of the total rejection are in agreement with the theoretical performance

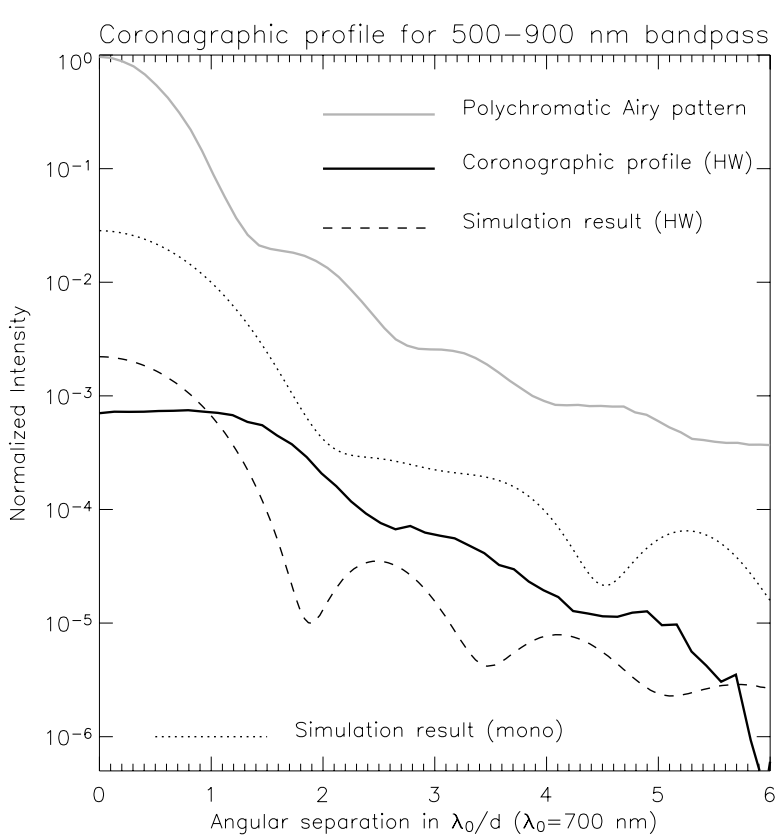

Fig. 5. Experimental and theoretical coronagraphic profiles: the grey solid curve is the experimental PSF for the 500-900 nm bandpass. The total exposure time for the PSF is $60 \mathrm{~s}$. The continuous black line shows the coronagrapic profile obtained with our achromatic waveplate mask. The coronagraphic image was obtained adding forty exposures of $30 \mathrm{~s}$ each. The dashed line presents the coronagraphic simulation results taking into account the waveplate phase residuals, the defocus error, the spectral response of the halogen lamp and the camera. For comparison, the dotted line presents the simulation results for a monochromatic mask used in the same conditions. All curves are azimuthally averaged (for this reason, the on-axis attenuation seems to be greater than the measured peak attenuation value of 755).

(see Sect. 2.4). In Fig. 6, we notice, in agreement with the coronagraphic profile of Fig. 5, that the residual level of $10^{-4}$ is quickly reached at $2.5 \lambda / d$.

The shape of the polychromatic coronagraphic image (Fig. 6) is similar to the classical FQPM case except that it is somewhat blurred compared to the monochromatic one (Riaud et al. 2003). To understand the blurring effect due to the large bandwidth $(R \approx 1.75)$, we simulated a polychromatic PSF passing through the coronagraph in order to quantify the residuals. In the Fourier transform calculations, we took into account the size of the pupil for each wavelength as well as the diameter of the Lyot stop in the relayed pupil. The dashed line in Figs. 5 and 7 presents simulation results taking into account the cumulative effects of the halfwave plates phase residuals, defocus errors induced by the use of simple lenses (no achromats) and the presence of a small spider on the mask transitions. For comparison, the dotted line presents the simulation results for a monochromatic mask used in the same conditions.

Given the spectral response of our Halogen lamp and bandpass filter, the calculated total rejection of the achromatic coronagraphic device for the bandpass $500-900 \mathrm{~nm}$ is 340 and the expected attenuation on the stellar peak is roughly 450 . The first value (total) is not far from measurements (294) whereas the second one (peak) is better in practice (755). For comparison, the theoretical total rejection for the monochromatic mask 


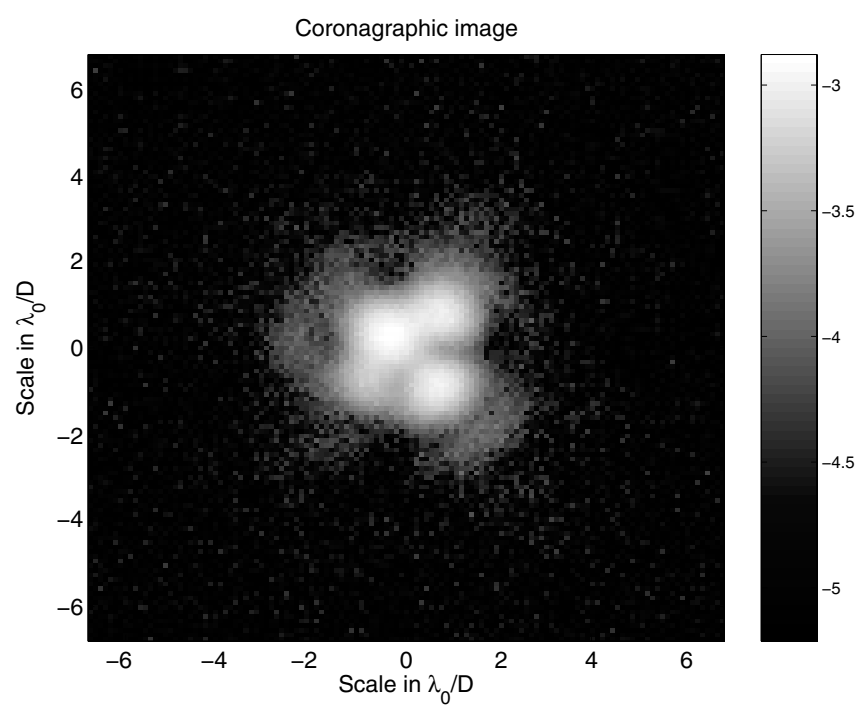

Fig. 6. Polychromatic coronagraphic residual image (logarithmic scale) for 500-900 nm bandpass. We use a 77\% Lyot stop for spatial filtering. The angular resolution is given for $\lambda_{0}=700 \mathrm{~nm}$ and for the entrance pupil (full pupil without diaphragm). The classical FQPM four-spot pattern is easily recognizable though somewhat blurred.

in the 500-900 $\mathrm{nm}$ range is only 35 . The discrepancy between the simulated and measured peak attenuation value could be explained by complex Fresnel diffraction effects induced by the spider intended to mask cutting imperfections. Indeed, classical Fourier propagation is not able to reproduce the four-spot shape of the actual broadband coronagraphic image (see Fig. 6). It is to be noted that the small width of the spider wires $(18 \mu \mathrm{m}$ in diameter corresponds to $\lambda / 7 d$ at $\lambda=500 \mathrm{~nm}$ ) does not affect the optical throughput nor the inner working distance.

The ratio between the two values (peak and total) is low in the polychromatic case $(\approx 1.32)$ compared to previous ratio obtained in the narrow band case with a laser diode $(\approx 10$, see Riaud et al. 2003). This observation is directly related to the polychromatic blurring effect.

For an actual stellar source, the rejection factor could be more important, depending on the maximum emissivity of the star. For example with the proposed 500-900 $\mathrm{nm}$ achromatization, a $\mathrm{G} 2 \mathrm{~V}$ and a $\mathrm{M} 2 \mathrm{~V}$ type stars would give better rejection factors. Indeed the maximum black body emissivity for these stars coincides with the two points of exact $\pi$ phase shift (see Fig. 3).

It is interesting to compare the results of our achromatic FQPM to the polarization interferometric coronagraph proposed by Baba et al. (2002). The peak attenuation is 6.5 better in our case but this value has to be balanced because of our slightly smaller bandpass $(500-900 \mathrm{~nm}$ compared to $370-830 \mathrm{~nm}$ ). It must also be noted that the throughput of the experiment presented in Baba et al. (2002) is limited by the two polarizers to only $<25 \%$.

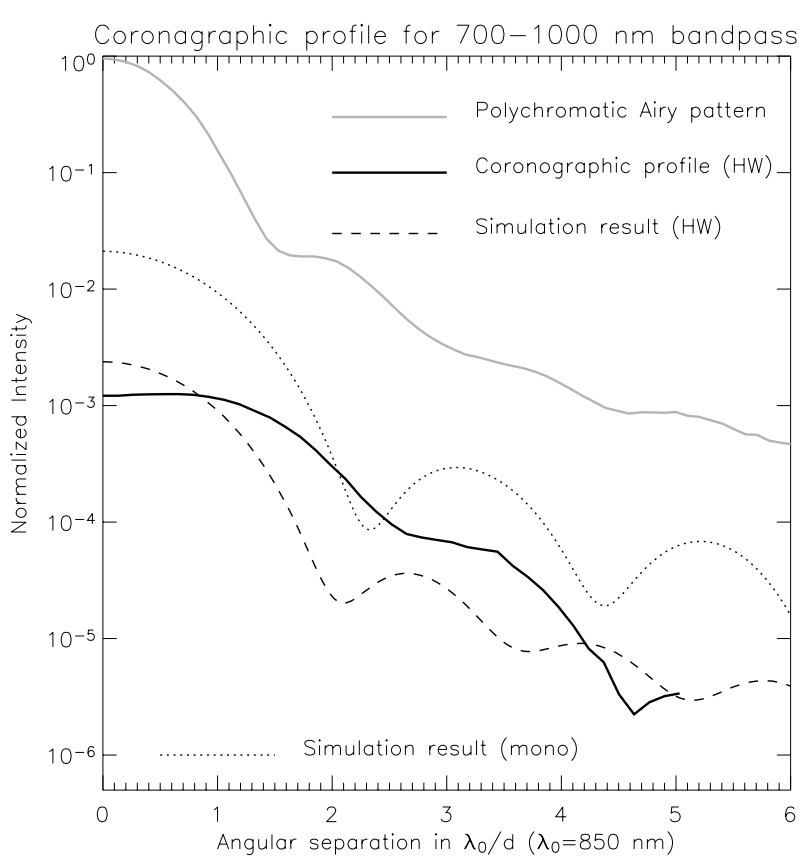

Fig. 7. Experimental and theoretical coronagraphic profiles: the grey solid curve is the experimental PSF for the $700-1000 \mathrm{~nm}$ bandpass. The total exposure time for the PSF is $60 \mathrm{~s}$. The continuous black line shows the coronagrapic profile obtained with our achromatic waveplate mask. The coronagraphic image was obtained adding forty exposures of $30 \mathrm{~s}$ each. The dashed line presents the coronagraphic simulation results taking into account the waveplate phase residuals, the defocus error, the spectral response of both the halogen lamp and the camera. For comparison, the dotted line presents the simulation results for a monochromatic mask used in the same conditions. All curves are azimuthally averaged (for this reason, the on-axis attenuation seems to be greater than the measured peak attenuation value of 561).

\section{Perspectives}

\subsection{Near infrared}

In the framework of the second generation instrumentation for the VLT (VLT-Planet Finder project) and following the results presented hereabove, we have proceeded with the manufacturing of near infrared components, using the same birefringent combination. Indeed, the Quartz transparency is well suited for wavelength ranges up to $2.6 \mu \mathrm{m}$ and $\mathrm{MgF}_{2}$ is transparent up to $8.5 \mu \mathrm{m}$. In Table 1, we present three different optimizations in the range $700-2500 \mathrm{~nm}$. The first one which covers the whole spectral range was assembled and will be tested soon. The large spectral range is achieved at the cost of a lower rejection factor. However, this component is well adapted to an instrument like NACO for which Strehl ratios of 50\% are routinely obtained.

The situation is quite different for VLT-PF, an instrument specifically optimized to image and characterize EGPs. For that, VLT-PF will include several coronagraphs each dedicated to a specific set of filters. The principle of detection relies on the Simultaneous Differential Imaging (SDI) method which consists in subtracting images taken simultaneously at different wavelengths (Marois et al. 2005). Such an instrument is already included in NACO although without coronagraphic capabilities (Hartung et al. 2004). This method provides self 
Table 1. Halfwave plate achromatization for various wavelength ranges from the $V$ band up to the $N$ band. The table summarizes the optimized thicknesses for the chosen doublet materials. The last line gives the total rejection factor.

\begin{tabular}{cccccc}
\hline \hline Filters & $500-900 \mathrm{~nm}(R=1.75)$ & $700-2500 \mathrm{~nm}(R=0.9)$ & $H(R=4.7)$ & $K(R=5.5)$ & $N(R=4.8)$ \\
\hline Couple $(\mathrm{a} / \mathrm{b})$ & & Quartz/MgF & & $\mathrm{CdS} / \mathrm{CdSe}$ \\
$d_{\mathrm{a}}(\mu \mathrm{m})$ & 841.1 & 1488.7 & 1332.2 & 1219 & 1798.1 \\
$d_{\mathrm{b}}(\mu \mathrm{m})$ & 674.7 & 1168 & 1057.9 & 977.1 & 1618.8 \\
$\tau$ (total rejection) & 340 & 260 & 7900 & $1.47 \times 10^{5}$ & $2.16 \times 10^{5}$ \\
\hline
\end{tabular}

reference subtraction while minimizing speckle residuals. It is expected to significantly improve the direct detection of EGPs and brown dwarfs. Basically, two or more wavelengths are observed simultaneously like for instance in the $H$ band: 1.575 , 1.600 and $1.625 \mu \mathrm{m}$. The $1.625 \mu \mathrm{m}$ line corresponds to the methane feature which, if present, shall be revealed by subtraction (stars have no methane signature contrary to low mass objects). In the $K$ band, the same method could also determine EGP $\mathrm{CO}_{2} / \mathrm{CH}_{4}$ proportions, which would constrain models of their atmospheres (Burrows et al. 2004; Chabrier et al. 2004; Sudarsky et al. 2003). However, the SDI method requires very low phase errors for proper subtraction of the adjacent wavelength narrow band images. For that reasons, we have searched for optimal solutions in the $H$ and $K$ bands and the results are presented in Table 1. Larger rejection rates are clearly feasible when the spectral range is narrower since the chromatic phase residual is also reduced.

\subsection{Towards the thermal infrared}

Earth-like planet detection is optimal at the planet's maximum emissivity, i.e. in the thermal infrared, around $12 \mu \mathrm{m}$. One of the most relevant biosignature is the strong Ozone spectral feature (DesMarais et al. 2003), around $9.65 \mu \mathrm{m}$. For this reason, the $N$ band appears to be a well-suited choice.

Infrared nulling interferometry (Bracewell 1978), considered for space-based missions like DARWIN/TPF (Léger et al. 1996) or ground-based experiments like Keck-I (Serabyn et al. 2004), is an interesting technique to achieve both high angular resolution and high contrast detection/characterization of exoplanets. This technique consists in adjusting the phases of the beams coming from various telescopes (two in the most simple configuration) to produce a pupil plane destructive interference on the optical axis. The phase shift ( $\pi$, for instance) may be provided in broad bands thanks to achromatic HWP.

An alternative to nulling interferometry is to recombine coherently all the telescopes in a single image plane. The HWP achromatic FQPM should then be regarded as an interesting solution if implemented at the Fizeau or densified focus of such an interferometer (Riaud et al. 2002). This approach would necessitate a minimum of three telescopes to be efficient (the main limitation of this method would be the cross-talk between sub-pupils). Preliminary numerical simulations show starlight attenuations of more than $10^{6}$ for a dilution factor (ratio of the interferometer baseline to the sub-pupil diameter) greater than 15 (Riaud et al. in preparation).

However, in the mid-infrared domain, the lack of birefringent materials would be the major problem for both approaches. Moreover, the few ones that can be found have large refractive indices, thereby inducing more losses due to spurious Fresnel reflections. Nonetheless, the couple $\mathrm{CdS} / \mathrm{CdSe}$ is a good candidate to perform achromatization optimizations in the thermal infrared. The phase shift residuals for this doublet with optimized thicknesses give a total rejection $\tau=2.1 \times 10^{5}$ for a theoretical spectral resolution $R=2.75$ in the $N$ band $(9-13 \mu \mathrm{m}$, see Table 1$)$. If a third material was incorporated, the equivalent total rejection could reach $10^{6}$. As said before, there are not many candidates, but a solution with $\mathrm{AgGaSe}_{2}$, for instance, is feasible.

\subsection{Artificially birefringent waveplates}

Another solution to achromatize the phase shift of the FQPM relies in the subwavelength grating technology. The period of subwavelength gratings is smaller than the wavelength of the incident light. They do not diffract light as classical spectroscopic gratings in the sense that only the zeroth transmitted and reflected orders are allowed to propagate outside the grating region, leaving incident wavefronts free from any further aberrations. For this reason, they are often called Zeroth Order Gratings (ZOGs). ZOGs present very specific properties: 1-D modulated ZOGs artificially create unique anisotropic and dispersive characteristics which can be used to synthesize achromatic waveplates from isotropic materials. This phenomenon is referred to as form birefringence. Two extensive studies on the implementation and optimization of such structures in coronagraphy were recently presented (Mawet et al. 2005a; Mawet et al. 2005b). Results of these studies are very promising with total rejections larger than $10^{5}$ for usual astrophysical bandfilters. The ZOG technology does not require any delicate assembly since the four gratings are engraved on a unique substrate. This solution is monolithic and therefore much more thermally stable. Furthermore, this technology is sufficiently flexible to accommodate a great variety of dielectric materials and is very well adapted to mid-infrared wavelength ranges.

\section{Conclusions}

We have demonstrated the feasibility of the FQPM coronagraph achromatization by means of birefringent elements. The halfwave plates technique was proved more convenient both to manufacture and to implement than the dispersive achromatization such as the dispersive plate concept (Mieremet et al. 2000). This conclusion comes directly from the fact that the fundamental constraint on the thickness control is relaxed by a factor comparable to the ratio between material indices and 
birefringences (often $<10^{-2}$ ) leading to a control at the $\approx 100 \mathrm{~nm}$ level versus $1 \mathrm{~nm}$ with the dispersive plate scheme.

In our experiment, we selected the Quartz- $\mathrm{MgF}_{2}$ doublet for application up to the near infrared domain on ground-based telescopes (until $2.6 \mu \mathrm{m}$ ). Results in white light are promising: we obtained a residual level of $10^{-4}$ at $2.5 \lambda / d$. The chromatic residue is sufficiently small to allow the use of this plate stack on a very large spectral range from the $R$ band to the $K$ band for moderate Strehl ratios. We also propose this type of achromatic mask optimized for the ESO's VLT-Planet Finder project. Theoretical results are well within its specifications and the experimentation in the visible has confirmed the feasibility and the interest of the technique.

We also expect to manufacture a monolithic component made of artificially birefringent waveplates using the more flexible ZOG technology. This would open further possibilities in high contrast imaging for ground/space-based facilities like the VLT-PF and TPF/DARWIN projects.

Acknowledgements. D.M. acknowledges the financial support of the Belgian "Fonds pour la formation à la Recherche dans l'Industrie et dans l'Agriculture". P.R. acknowledges the financial support of the "Pôle d'Attraction Inter-Universitaire". We also are very grateful to the financial support of the "BQR" contract from the Paris-Meudon Observatory. We warmly acknowledge Olivier Absil and the anonymous referee for useful comments.

\section{References}

Baba, N., Murakami, N., Ishigaki, T., \& Hashimoto, N. 2002, Opt. Lett., 27, 1373

Boccaletti, A., Riaud, P., Baudoz, P., et al. 2004, PASP, 116, 1061

Bracewell, R. N. 1978, Nature, 274, 780
Burrows, A., Sudarsky, D., \& Hubeny, I. 2004, ApJ, 609, 407

Chabrier, G., Allard, F., Baraffe, I., Barman, T., \& Hauschildt, P. H. 2004, in Extrasolar Planets: Today and Tomorrow, ed. J.P. Beaulieu, A. Lecavelier des Etangs, \& C. Terquem, Proc. ASP Conf., 321, 131

DesMarais, D. J., Lin, D., Harwit, M., et al. 2001, Biosignatures and Planetary Properties to be Investigated by the TPF Mission, JPL Publication 01-008, California Inst. Of Technology, Pasadena, CA, 57

Frecker, J. E., \& Serkowski, K. 1976, Appl. Opt., 15, 605

Hariharan, P. 1996, Opt. Engineering, 35, 3335

Hartung, M., Herbst, T. M., Close, L. M., et al. 2004, A\&A, 421, L17

Léger, A., Mariotti, J. M., Mennesson, B., et al. 1996, Icarus, 123, 249

Marois, C., Doyon, R., Nadeau, D., et al. 2005, PASP, 117, 745

Mawet, D., Riaud, P., Absil, O., \& Surdej, J. 2005a, ApJ, 633, 1191

Mawet, D., Riaud, P., Surdej, J., \& Baudrand, J. 2005b, Appl. Opt., 44, 7313

Mieremet, A. L., Braat, J. J., Bokhove, H., \& Ravel, K. 2000, in Interferometry in Optical Astronomy, ed. P. J. Léna, \& A. Quirrenbach, Proc. SPIE, 4006, 1035

Mouillet, D., Fusco, T., Lagrange, A.-M., \& Beuzit, J.-L. 2003, EAS Pub. Ser., 8, 193

Riaud, P., Boccaletti, A., Rouan, D., Lemarquis, F., \& Labeyrie, A. 2001, PASP, 113, 1145

Riaud, P., Baudrand, J., Boccaletti, A., \& Rouan, D. 2003, PASP, 115, 712

Rouan, D., Riaud, P., Boccaletti, A., Clénet, Y., \& Labeyrie, A. 2000, PASP, 112, 1479

Rousset, G., Lacombe, F., Puget, P., et al. 1998, in Adaptive Optical System Technologies, ed. D. Bonaccini, \& R. K. Tyson, Proc. SPIE, 3353, 508

Serabyn, E., Booth, A. J., Colavita, M. M., et al. 2004, in New Frontiers in Stellar Interferometry, ed. W. Traub, Proc. SPIE, 5491, 806

Sudarsky, D., Burrows, A., \& Hubeny, I. 2003, ApJ, 588, 1121 